\title{
Como estão as condições socioeconômicas, de saúde e de trabalho de idosos trabalhadores e aposentados?
}

\author{
How are the socioeconomic, health and working conditions of older workers and retirees? \\ ¿Cómo son las condiciones socioeconómicas, sanitarias y laborales de los trabajadores mayores y jubilados?
}

\begin{abstract}
RESUMO
Objetivo: Conhecer as condições socioeconômicas, de saúde e de trabalho de idosos jovens. Método: Estudo prospectivo, exploratório e descritivo desenvolvido com idosos entre 60 e 70 anos, ou seja, idosos jovens. As entrevistas aconteceram entre novembro de 2018 e janeiro de 2019. Os dados coletados foram armazenados, tabulados em planilha do Microsoft Excel ${ }^{\circledR}$ e analisados por meio de estatística descritiva. Resultados: Participaram 44 idosos jovens com funcionalidade, cognição e humor preservados, majoritariamente feminina, com boa autopercepção de saúde e de capacidade para o trabalho. Além disso, eram idosos com hábitos de vida saudáveis, que participavam de diferentes atividades sociais e de lazer. Conclusão: Os idosos se configuram um grupo geracional heterogêneo, no qual é importante conhecer informações sobre as condições de vida, saúde e trabalho para nortear políticas públicas e atender suas necessidades e interesses.
\end{abstract}

DESCRITORES: Idoso; Trabalho; Aposentadoria.

\section{ABSTRACT}

Objective: To know the socioeconomic, health and work conditions of young elderly people. Method: Prospective, exploratory and descriptive study developed with elderly people between 60 and 70 years old, that is, young elderly people. The interviews took place between November 2018 and January 2019. The collected data were stored, tabulated in a Microsoft Excel ${ }^{\circledR}$ spreadsheet and analyzed using descriptive statistics. Results: 44 young elderly people with preserved functionality, cognition and mood participated, mostly female, with good self-perception of health and ability to work. In addition, they were elderly people with healthy lifestyle habits, who participated in different social and leisure activities. Conclusion: The elderly are a heterogeneous generational group, in which it is important to know information about living, health and work conditions to guide public policies and meet their needs and interests.

DESCRIPTORS: Elderly; Job; Retirement.

\section{RESUMEN}

Objetivo: Conocer las condiciones socioeconómicas, de salud y laborales de los jóvenes adultos mayores. Método: Estudio prospectivo, exploratorio y descriptivo desarrollado con personas mayores entre 60 y 70 años, es decir, jóvenes ancianos. Las entrevistas se llevaron a cabo entre noviembre de 2018 y enero de 2019. Los datos recopilados se almacenaron, tabularon en una hoja de cálculo de Microsoft Excel ${ }^{\circledR}$ y se analizaron mediante estadística descriptiva. Resultados: participaron 44 jóvenes adultos mayores con funcionalidad, cognición y estado de ánimo conservados, en su mayoría mujeres, con buena autopercepción de la salud y capacidad para trabajar. Además, se trataba de personas mayores con hábitos de vida saludables, que participaban en diferentes actividades sociales y de ocio. Conclusión: Los adultos mayores son un grupo generacional heterogéneo, en el que es importante conocer información sobre las condiciones de vida, salud y trabajo para orientar las políticas públicas y atender sus necesidades e intereses. É necessário fornecer o texto original para ver mais informações sobre a tradução

DESCRIPTORES: Anciano; Trabajo; Jubilación.

RECEBIDO EM: 30/03/2021 APROVADO EM: 03/05/2021

\section{João Henrique de Morais Ribeiro}

Enfermeiro. Doutor em Ciências pela Escola de Enfermagem da Universidade de São Paulo (EEUSP). Docente da Faculdade de Enfermagem da Universidade Santo Amaro (UNISA).

ORCID: 0000-0002-6411-8839 


\section{Barbara Bartuciotti Giusti}

Psicóloga. Doutoranda do Programa Interunidades em Enfermagem da Escola de Enfermagem e da Escola de Enfermagem de Ribeirão Preto da USP.

ORCID: 0000-0002-2716-801X

\section{Suely Itsuko Ciosak}

Enfermeira. Professora Associada 3 do Departamento de Enfermagem em Saúde Coletiva da Escola de Enfermagem da USP. ORCID: 0000-0001-5884-2524

\section{INTRODUÇÃO}

$\mathbf{N}$ o que se refere ao binômio envelhecimento e desenvolvimento econômico, um influenciará o outro de maneira abrangente e social. Pesquisa na área econômica considera que o envelhecimento da população aumenta a escassez de mão de obra e pode resultar em redução na produtividade e produção de bens e serviços, acarretando a queda da poupança total e diminuição da acumulação de capital, com consequente retração no crescimento econômico ${ }^{1}$.

No cenário laboral relacionado à população idosa, Wajnman, Oliveira, Oliveira $(2004)^{2}$ afirmaram que o aumento de trabalhadores acima de 50 anos pressionou o mercado de trabalho brasileiro na perspectiva de geração de postos de trabalho e, conforme a Pesquisa Mensal de Empregos realizada em 2013 foram registrados aumentos nesta faixa etária, sobretudo para as pessoas acima de 65 anos, na qual houve aumento de $11,66 \%$ nos postos de trabalho relacionado ao avanço no grau de instrução desses trabalhadores ${ }^{3}$.

A Pesquisa Nacional por Amostra de Domicílios Contínua do primeiro trimestre de 2017 revelou que os idosos representam $7,3 \%$ das pessoas ocupadas, com maior participação nas regiões Sudeste e Sul. Na comparação entre 2016 e 2017, as pessoas com 60 anos e mais apresentavam tendência de aumento na participação na população ocupada4. Essa tendência foi confirmada no segundo semestre de 2018, revelando que $7,9 \%$ dos idosos brasileiros estão ocupados 5 .

Assim é possível perceber que, cada vez mais, idosos estarão no mercado de trabalho, distanciando-se do imaginário social que espera, de forma geral, que a chegada dos 60 anos seja sinônimo de afastamento do mundo laboral e consequente aposentadoria ${ }^{6}$.

A necessidade de complementar a renda pessoal ou familiar, a manutenção das relações sociais, bem como a motivação individual, tem incentivado os idosos a permanecerem ou se reinserirem no mercado de trabalho, possibilitando novos aprendizados e valorização de suas experiências profissionais, reduzindo o déficit causado pelo sistema previdenciário?.

A Organização Mundial da Saúde propôs a Estratégia Global e Plano de Ação sobre o Envelhecimento e a Saúde com ações que fomentam o envelhecimento saudável e o fortalecimento nacional para formulação de políticas baseadas em evidências que possam combater o preconceito relacionado a idade avançada ${ }^{8}$. Para tal, faz-se necessário atender as necessidades desse grupo etário, desenvolvendo políticas organizacionais e de recursos humanos capazes de promover um ambiente de trabalho saudável e livre de discriminação.

Para se viver de forma mais saudável, o trabalho deve ser considerado um ponto importante na longevidade, pois é nesse contexto que os sujeitos, muitas vezes, encontram seu lugar social. Consequentemente tem-se ampliado as discussões a respeito do tempo ocioso que pode ser percebido com a chegada dos 60 anos e com a aposentadoria, discussões estas que perpassam a reforma da previdência e o aumento da idade mínima para a efetivação da aposentadoria, tema atual na sociedade como um todo.

Assim, emergiu a questão que norteia este estudo: Como estão as condições socioeconômicas, de saúde e trabalho de idosos trabalhadores e aposentados?

Acredita-se que essa temática deva ser explorada, pois desmistifica o idoso como um sujeito que pode onerar o desenvolvi- mento de países e dá visibilidade as suas potencialidades como um ser social engajado com o trabalho.

O objetivo deste estudo foi conhecer as condiçõos socioeconômicas, de saúde e de trabalho de idosos jovens.

\section{MÉTODO}

Estudo prospectivo, exploratório e descritivo desenvolvido com idosos entre $60 \mathrm{e}$ 70 anos, ou seja, idosos jovens.

O formulário utilizado para a coleta de dados foi elaborado de forma colaborativa entre os pesquisadores, composto por 20 questóes que versavam sobre as características socioeconômicas e a condição de saúde dos participantes. $\mathrm{O}$ instrumento foi testado previamente para otimizar a coleta e reduzir possíveis erros de compreensão.

Para conhecer a capacidade funcional e cognitiva dos participantes, bem como a presença de quadro depressivo foram empregados três instrumentos consagrados e validados, os quais compõem a Avaliação Multidimensional do Idoso, sendo o Mini Exame do Estado Mental (MEEM), a Escala de Lawton e a Escala de Depressão Geriátrica (EDG 15 itens). No Brasil, há diferentes versões e pontos de corte desses instrumentos e adotou-se, neste estudo, as versões dos instrumentos e as notas de corte propostas pelo Ministério da Saúde9.

Os participantes foram localizados em espaços públicos, domicílios, locais de trabalho e selecionados por conveniência. Após anuência em participar, os pesquisadores agendaram data e horário para a realização das entrevistas.

As entrevistas foram realizadas entre novembro de 2018 e janeiro de 2019, utilizando o formulário descrito acima e aconteceram na residência dos participantes. 


\section{artigo}

Ribeiro, J.H.M.; Giusti, B.B.; Ciosak, S.I.

Como estão as condições socioeconômicas, de saúde e de trabalho de idosos trabalhadores e aposentados?

Foram incluídos idosos jovens que atenderam os seguintes critérios de inclusão: ambos os sexos, aposentados ou não e que concordaram em participar do estudo através da leitura e assinatura do Termo de Consentimento Livre e Esclarecido (TCLE). Foram excluídos idosos com in- capacidade, como os acamados, com limitações funcionais graves e aqueles que se recusaram a participar do estudo.

Os dados coletados foram armazenados e tabulados em planilha do Microsoft Excel $^{\circ}$, sendo analisados por meio de estatística descritiva na qual as questões quali-

Tabela 01 - Características socioeconômicas dos idosos jovens segundo localidade. São Paulo, 2019.

\begin{tabular}{|c|c|c|c|c|c|}
\hline \multirow{2}{*}{ VARIÁVEL } & \multicolumn{2}{|c|}{ TOTAL } & \multirow{2}{*}{ VARIÁVEL } & \multicolumn{2}{|c|}{ TOTAL } \\
\hline & $\mathbf{N}$ & $\%$ & & $\mathbf{N}$ & $\%$ \\
\hline Faixa etária & & & Moradia & & \\
\hline 60 a 65 anos & 16 & 36 & Própria & 41 & 93 \\
\hline 66 a 70 anos & 28 & 64 & Alugada & 3 & 6,8 \\
\hline Sexo & & & Dependentes da renda & & \\
\hline Masculino & 14 & 32 & Nenhuma & 15 & 34 \\
\hline \multirow[t]{2}{*}{ Feminino } & 30 & 68 & 1 pessoa & 13 & 30 \\
\hline & & & 1 a 4 pessoas & 16 & 36 \\
\hline \multicolumn{6}{|l|}{ Estado civil } \\
\hline Solteiro & 1 & 2,3 & Arranjo Familiar & & \\
\hline Casado/União estável & 30 & 68 & Mora sozinho & 7 & 16 \\
\hline Separado/Divorciado & 8 & 18 & Com o esposo & 20 & 45 \\
\hline Viúvo & 5 & 11 & Com os filhos & 6 & 14 \\
\hline \multicolumn{6}{|l|}{ Religião } \\
\hline Católica & 39 & 89 & Participação social & & \\
\hline Evangélica & 2 & 4,5 & & & \\
\hline Espírita & 2 & 4,5 & Sim & 36 & 82 \\
\hline Outras & 1 & 2,3 & Não & 8 & 18 \\
\hline \multicolumn{6}{|l|}{ Escolaridade } \\
\hline 1 a 3 anos de estudo & 3 & 6,8 & & & \\
\hline 4 a 7 anos de estudo & 5 & 11 & & & \\
\hline Mais de 7 anos & 36 & 82 & & & \\
\hline TOTAL & & & & 44 & 100 \\
\hline Fonte: Os autores, 2019. & & & & & \\
\hline
\end{tabular}

Tabela 02 - Condição de trabalho e ocupação dos idosos jovens segundo localidade. São Paulo, 2019

$$
\text { VARIÁVEL }
$$

TOTAL

\section{Situação atual de trabalho}

Trabalho formal

Trabalho informal/voluntário

Aposentado

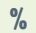

77,3 tativas estão exibidas em valores absolutos (n) e relativos (\%) e as quantitativas expressas por meio das medidas de posição e dispersão, ambos apresentados em tabelas.

Atendendo a Resolução 466/2012, este estudo foi submetido e aprovado pelo Comitê de Ética em Pesquisa da Escola de Enfermagem da Universidade de São Paulo com o parecer n ${ }^{\circ}$ CAAE 79330817.3.0000.5392.

\section{RESULTADOS}

Participaram 44 idosos, e a maioria apresentou escores satisfatórios para os três testes Em relação ao MEEM, 30,5\% $(\mathrm{n}=11)$ dos idosos com mais de sete anos de estudo apresentaram escore inferior ao relacionado à sua escolaridade, indicando risco para declínio cognitivo, sendo três idosos do sexo masculino e oito do feminino, com idade entre 64 e 70 anos. Dentre esses idosos, 13,9\% ( $n=5)$ encontravam-se em atividades laborais formais.

Já a funcionalidade, aferida por meio das atividades instrumentais de vida diária, os idosos se mostraram com capacidade preservada, entre os quais $84,1 \%$ atingiram a pontuação máxima do instrumento.

Apenas uma idosa, com 60 anos e mais e de setes anos de escolaridade apresentou indicativo de depressão leve.

A idade média foi de 66,2 anos (dp: 3,36 ), destes $63,6 \%$ estavam entre 66 e 70 anos. $68,2 \%$ eram do sexo feminino e $68,2 \%$ casados. Quanto ao arranjo familiar, a maior parte $(84 \%)$ moravam com o esposo e filhos, compartilhando a renda com até quatro pessoas (36,4\%), 16\% moravam sozinhos e $34,1 \%$ não declaravam dependentes da renda. Pode-se perceber que a participação em atividades sociais (81.8\%) é frequente (Tabela 01).

Quanto a situação de trabalho, a maioria era aposentado $(77,3 \%)$ e $18,2 \%$ continuavam em atividades laborais formais. Além disso, 54,6\% dos idosos referiram boa capacidade para o trabalho (Tabela 02).

Em relação às condições de saúde, $70,5 \%$ percebia sua saúde como boa e a maioria $(81,8 \%)$ não consumiam tabaco 


$\begin{array}{lcc}\text { Capacidade de trabalho autorreferida } & \\ \text { Ótima } & 8 & 18,2 \\ \text { Boa } & 24 & 54,6 \\ \text { Moderada } & 10 & 22,7 \\ \text { Ruim } & 2 & 4,5 \\ \text { Faltas ao trabalho } & & \\ \text { Não } & 44 & 100 \\ \text { TOTAL } & 44 & 100 \\ \text { Fonte: Os autores, 2019. 'Os idosos que realizavam trabalho informal/voluntário jáse encontravam aposentados. } & \end{array}$

Tabela 03 - Condição de saúde de idosos jovens segundo localidade. São Paulo, 2019

VARIÁVEL

TOTAL

N

$\%$

Auto percepção de saúde

Muito boa

11,3

Boa

70,5

Regular

Consumo de tabaco

Sim

Não

Consumo de bebida alcoólica

Sim

Não

Plano de saúde

Sim

Não

Uso diário de medicamentos

Sim

Não

Quantidade diária ingerida

1 a 2 medicamentos

3 a 4 medicamentos

5 a 6 medicamentos

Não faz uso

Aquisição dos medicamentos

Sistema Único de Saúde

Doação de terceiros

Compra com recurso próprio

63,6

Não faz uso

Internação hospitalar no último ano

Sim e $75 \%$ não faziam uso de bebidas alcóolicas, a mesma porcentagem tinham plano de saúde. Houve predomínio (90,9\%) dos que faziam uso diário de medicamentos. (Tabela 03).

Apesar da maioria ser aposentado $63,6 \%$ dos idosos adquiriam os medicamentos com seus próprios recursos e 36,4\% no Sistema Único de Saúde (SUS). Somente $13,6 \%$ dos idosos foram hospitalizados no último ano e $52,3 \%$ não realizaram tratamento odontológico (Tabela 03).

Quanto a quedas, 72,73\% informaram não ter caído no último ano, porém verificou-se entre as ocorrências de queda.

\section{DISCUSSÃO}

Entre os idosos jovens participantes desse estudo, a idade média foi 66,2 anos (dp: 3.36), média de idade semelhante às encontradas em estudos realizados com moradores da zona rural mineira ${ }^{10}$ e em outros países como China e Cingapura ${ }^{11}$.

Com o aumento da expectativa de vida, o número de idosos no mercado de trabalho sofreu um acréscimo significativo e de acordo com as projeções para 2040, cerca de $57 \%$ da população terá idade superior a 45 anos. Portanto, mais pessoas continuarão em suas atividades laborais gerando dificuldades de pagamento das aposentadorias $^{12}$ e favorecendo posturas discriminatórias baseadas na idade ${ }^{13}$.

Algumas organizações têm empregado uma ferramenta denominada Gestão da Idade que tem como finalidade a adoção de uma postura positiva frente ao envelhecimento, com o intuito de oferecer propostas e reduzir as barreiras etárias ${ }^{14}$.

Grande parte dos participantes desse estudo era do sexo feminino $(68,2 \%)$ corroborando com outro estudo na qual havia a maior participação de mulheres idosas $^{15}$. Estudos denotam essa prevalência em todo mundo e tem despertado os olhares da sociedade para o fenômeno denominado feminização da velhice ${ }^{16,17}$.

A discriminação pela idade, a renda insuficiente, o isolamento social, a aposentadoria, viuvez, afastamento dos filhos e o 
Não

\section{Quedas no último ano}

Sim

Não

TOTAL

Fonte: Os autores, 2019.
38 12

32

44
86,4

27,3

72,7

100 cuidado aos familiares dependentes exercem influência nas idosas o que as tornam mais vulneráveis ${ }^{18}$.

Quanto ao estado civil, o perfil encontrado é similar em estudos com idosos americanos ${ }^{19}$ e com trabalhadores petroquímicos no Irã ${ }^{20}$. Há evidências ${ }^{19}$ de que a parceria entre casais pode ser benéfica no contexto do trabalho, favorecendo novas perspectivas e canais abertos de emprego entre os casais em que um dos cônjuges se encontra no ambiente laboral. Outro estudo relata, que a influência do parceiro pode favorecer na decisão pela aposentadoria precoce ${ }^{21}$.

De acordo com o Censo de 2010, $87,2 \%$ das famílias brasileiras eram compostas por duas ou mais pessoas com um grau de parentesco e $12,1 \%$ viviam sozinhas $^{22}$, o que se aproxima dos resultados deste estudo.

A família pode ser considerada um dos principais eixos da sociedade, desempenhando papel central na economia, além de ser fonte de proteção, solidariedade, indispensáveis para a sobrevivência ${ }^{22,23}$.

$\mathrm{Na}$ contemporaneidade, a família vem sendo o centro do debate político pois, com o envelhecimento populacional, já é possível observar a constituição de novos arranjos familiares denominados famílias intergeracionais ${ }^{24}$ Em meio a essas mudanças, o idoso passa a desempenhar novos papéis, o de provedor do lar. No Brasil, os rendimentos dos idosos, muitas vezes derivados de sua aposentadoria e pensões, tem sido identificada como a principal fonte de renda da família, especialmente dos mais jovens ${ }^{25,26}$.

Entre os determinantes sociais, o envolvimento religioso tem um papel significativo e pode ampliar as condições para lidar com as perdas decorrentes do próprio envelhecer ${ }^{27}$. Houve predomínio do catolicismo e estudos sugerem que mesmo diante do crescimento de novas religiões, a população brasileira mantém a hegemonia da católica ${ }^{22}$.

Outro fator relevante é o nível de escolaridade, uma vez que idosos com maior escolaridade apresentam melhores condições de saúde e uma ocupação mais qualificada que lhes possibilita melhor remuneração ${ }^{28,29}$. Diversas são as iniciativas públicas e ações não governamentais cuja finalidade é a de oferecer alfabetização e educação continuada para adultos e idosos dado o impacto da escolaridade na vida social, econômica e trabalhista ${ }^{30}$.

A autopercepção positiva da saúde foi encontrada em diferentes estudos: no sul do Brasil a prevalência dessa percepção foi de $51,2 \%{ }^{31}$, enquanto no estudo em Campinas $^{32}, 80,9 \%$ dos idosos percebiam positivamente sua condição de saúde. A autopercepção de saúde vem sendo empregada rotineiramente por ser vista como uma prática segura e confiável para predizer a sobrevida e expressar diferentes aspectos da saúde física, cognitiva, e emocional dos sujeitos ${ }^{33}$. Perceber melhor a saúde, estava associado ao consumo moderado de álcool, lazer, história de queda no último ano e a ausência de polifarmácia ${ }^{31}$, condiçõos semelhantes a encontradas em nossa população.

Com relação à participação em atividades de lazer, há necessidade de promover comportamentos e hábitos saudáveis a fim de apoiar as iniciativas de extensão da vida profissional entre os idosos ${ }^{34}$. Além disso, a promoção da participação em organizações religiosas, grupos de amizade e reuniōes familiares pode ajudar a preservar a função cognitiva dos idosos ${ }^{35}$.

Dentre os fatores que exercem influência na permanência de idosos no mercado de trabalho, as condições de saúde têm sido amplamente estudadas e apontadas como um dos principais determinantes para que isso ocorra ${ }^{36,37}$.

Quanto a situação atual de trabalho e sua capacidade autorreferida para trabalhar, a maioria dos idosos jovens encontrava-se aposentada e $18,2 \%$ estavam ativos nas atividades laborais, inclusive desempenhando trabalho voluntário. Ademais, percebiam sua capacidade para trabalhar como boa.

No estudo desenvolvido por Ribeiro et al. $(2018)^{25} 13,1 \%$ dos idosos mantinham atividade remunerada e, entre trabalhadores americanos. com idade média de 59 anos, $82 \%$ deles realizavam trabalho assalariado. O trabalho traz diferentes benefícios que vão desde a promoção da autoestima, a utilidade, os relacionamentos até a segurança financeira, tendo como um de seus determinantes o desejo de contribuir para a sociedade 25,38 .

Sendo assim, muitos idosos, aposentados ou não, continuam trabalhando por algum tipo de necessidade, seja ela financeira, psicológica ou social, sendo que essa opção está diretamente ligada ao significado que cada um atribui ao trabalho ${ }^{39}$.

\section{CONCLUSÃO}

Este estudo nos possibilitou conhecer o perfil socioeconômico de idosos entre 60 e 70 anos e suas condições de trabalho e saúde.

Quanto ao perfil socioeconômico e as condições de trabalho e saúde dos participantes encontrou-se uma população com funcionalidade, cognição e humor preservados, majoritariamente feminina, com boa autopercepção de saúde e de capacidade para o trabalho. Além disso, eram idosos com hábitos de vida saudáveis, que participavam de diferentes atividades sociais e de lazer.

Os idosos se configuram um grupo geracional heterogêneo na qual é importante conhecer informações sobre os aspectos socioeconômicos, demográficos, condições de saúde e estilo de vida, que poderão nortear políticas públicas, programas e modelos de intervenção adequados para atender suas necessidades e seus interesses ${ }^{46}$. 
É premente necessidade de investimentos em estratégias que garantam melhores condições e participação dos idosos no mercado de trabalho de acordo com sua vontade, livres de preconceito e exercendo sua dignidade e direitos.

\section{REFERÊNCIAS}

1. Haiming L, Zhang X. Population Aging and Economic Growth: The Chinese Experience of Solow Model. International Journal of Economics and Finance [Internet] 2015 [cited 2021 Apr 16]; 7(3): 199-206. Available from: http://www.ccsenet.org/journal/ index.php/ijef/article/view/43303.

2. Wajnman S, Oliveira AMHC, Oliveira EL. Os idosos no mercado de trabalho: tendências e consequências. In: Camarano AA (org.). Os novos idosos brasileiros: muito além dos 60 anos. [citado 2021 Abril 16]. Rio de Janeiro: IPEA; 2004. Disponivel em: http://repositorio.ipea.gov.br/handle/11058/3012.

3. Instituto Brasileiro de Geografia e Estatística. Síntese de indicadores sociais: uma análise das condições de vida da população brasileira [citado 2021 Abril 16]. Rio de Janeiro; 2013. Disponível em: http://biblioteca.ibge.gov.br/visualizacao/livros/liv66777.pdf.

4. Pesquisa Nacional por Amostra de Domicilio Contínua: primeiro trimestre de 2017. [citado 2021 Abril 16]. Brasilia, 2017. Disponível em: ftp://ftp.ibge.gov.br/Trabalho_e_Rendimento/Pesquisa_Nacional_por_Amostra_de_Domicilios_continua/Trimestral/Fasciculos_Indicadores_IBGE/pnadc_201701_trimestre_caderno.pdf

5. Pesquisa Nacional por Amostra de Domicílio Contínua: segundo trimestre de 2018. [citado 2021 Abril 16]. Brasília, 2018. Disponivel em: https://biblioteca.bge.gov.br/visualizacao/periodicos/2421/pnact_2018_2tri.pdf

6. Paolini KS. Desafios da inclusão do idoso no mercado de trabalho. Rev Bras Med Trab. [Internet] 2016 [citado 2021 Abril 16];14(2):177-82. Disponivel em: https://www.researchgate.net/ publication/310491234_Desafios_da_inclusao_do_idoso_no_ mercado_de_trabalho.

7. Queiroz VS, Ramalho HMB, Cavalcanti GM. 0 emprego do idoso no mercado de trabalho: evidências para o Brasil a partir da PNAD de 2005 [citado 2021 Abril 16]. Disponivel em: http://aplicativos. fipe.org.br/enaber/pdf/132.pdf.

8. World Health Organization. Global strategy and action plan on ageing and health. Geneva: World Health Organization; 2017. Licence: CC BY-NC-SA 3.0 IGO. Available from: https://www.who.int/ ageing/WHO-GSAP-2017.pdf

9. Brasil. Ministério da Saúde. Envelhecimento e saúde da pessoa idosa [citado 2021 Abril 16]. Brasilia: Ministério da Saúde, 2007. Disponivel em: http://bvsms.saude.gov.br/bvs/publicacoes/abcad19.pdf.

10. Garbaccio JL, Tonaco LAB, Estêvão WG, Barcelos BJ. Aging and quality of life of elderly people in rural areas. Rev. Bras. Enferm. [Internet]. 2018 [cited 2021 Apr 16] ; 71( Suppl 2 ): 724-732. Available from: http://www.scielo.br/scielo.php?script=sci_arttext\&pid=S0034-71672018000800724\&lng=en.

11. Schwingel A, Niti MM, Tang C, Pin Ng T. Continued work employment and volunteerism and mental well-being of older adults: Singapore longitudinal ageing studies. Age and Ageing. [Internet] 2009 [cited $2021 \mathrm{Apr} 16$ ]; 38(5): 531-537. Available from: https://academic.oup.com/ageing/article/38/5/531/2462137.
12. França LHFP, Siqueira-Brito AR, Valentini F, Vasques-Menezes I, Torres CV. Ageism in the organizational context - the perception of Brazilian workers. Rev. bras. geriatr. gerontol. [Internet]. 2017 [citado $2021 \mathrm{Abr} 16$ ] ; 20( 6): 762-772. Disponivel em: http://www.scielo.br/scielo.php?script=sci_arttext\&pid=S1809-98232017000600762\&lng=pt.

13. Goldani AM. Desafios do "preconceito etário" no Brasil. Educ. Soc. [Internet] 2010 [citado 2021 Abril 16]; 31(111): 411-434. Disponivel em: https://www.scielo.br/scielo.php?script=sci_arttext\&pid=S0101-73302010000200007 .

14. Cepellos VM. $O$ envelhecimento nas Organizações: das percepções de gestores de Recursos Humanos às práticas de gestão da idade. 2013. 98 f. Dissertação (Mestrado em Administração de Empresas) - Escola de Administração de Empresas [citado 2021 Abril 16]. Fundação Getúlio Vargas, São Paulo, 2013. Disponível em: https://pesquisa-eaesp.fgv.br/sites/gvpesquisa.fgv.br/files/ vanessa_martines_cepellos.pdf.

15. Lima Lobato TC, Araujo Reis D, de Souza Dantas J, Marinho de Souza DB, Dantas de Souza A, Garcia Siqueira G. Prevalência de doenças crônicas não transmissiveis em idosos longevos em um município no interior do Amazonas. Saúde Coletiva (Barueri) [Internet]. 2021 [citado 2021 Abril 16]; 11(63), 55085519. Disponivel em: https://doi.org/10.36489/saudecoletiva.2021v11i63p5508-5519.

16. United Nations Department of Economic and Social Affairs (UN/DESA). World Economic Situationand Prospects 2013 [cited 2021 Apr 16]. United Nations, New York, 2013. Available from: https:/www.un.org/development/desa/dpad/wp-content/uploads/sites/45/publication/2013wesp.pdf.

17. Fundo de População das Nações Unidas. Relatório sobre a situação da população mundial 2012 [cited 2021 Apr 16]. Disponivel em: http://www.unfpa.org.br/Arquivos/swop2012.pdf.

18. Lima LCV, Bueno CMLB. Envelhecimento e gênero: a vulnerabilidade de idosas no Brasil. Revista Saúde e Pesquisa [Internet] 2009 [citado 2021 Abril 16]; 2(2): 273-280. Disponivel em: https://periodicos.unicesumar.edu.br/index.php/saudpesq/article/view/1173.

19. Gonzales E, Nowell WB. Social Capital and Unretirement: Exploring the Bonding, Bridging, and Linking Aspects of Social Relationships. Research on Aging [Internet] 2017 [cited 2021 Apr 16]; 39(10), 1100-117. Available from: https://journals.sagepub.com/ doi/abs/10.1177/0164027516664569?rfr_dat=cr_pub\%3Dpubmed\&url_ver=Z39.88-2003\&rfr_id=ori\%3Arid\%3Acrossref. org\&journalCode=roaa.

20. Mazloumi A, Rostamabadi A, Saraji GN, Foroushani AR. Work Ability Index (WAI) and its association with phychosocial factors in one of the Petrochemical Industries in Iran. J Occup Health [Internet] 2012 [cited $2021 \mathrm{Apr} 16$ ]; 54(2), 112-118. Available from: https://pubmed.ncbi.nlm.nih.gov/22333439/. 


\section{REFERÊNCIAS}

21. Wind A, Geuskens GA, Ybema JF, Blatter BM, Burdorf $A$, Bongers PM, van der Beek AJ. Health, job characteristics, skills, and social and financial factors in relation to early retirement results from a longitudinal study in the Netherlands. Scand J Work Environ Health [Internet] 2014 [cited 2021 Apr. 16];40(2):186194. Available from: http://www.sjweh.fi/show_abstract.php?abstract_id=3393.

22. Instituto Brasileiro de Geografia e Estatística. Censo Demográfico 2010. Características gerais da população, religião e pessoas com deficiência [citado 2021 Mai.16]. Rio de Janeiro. IBGE, 2010. p. 31. Disponível em: http://biblioteca.ibge.gov.br/visualizacao/periodicos/94/cd_2010_religiao_deficiencia.pdf.

23. Rabelo DF, Neri AL. The household arrangements, physical and psychological health of the elderly and their satisfaction with family relationships. Rev. bras. geriatr. gerontol. [Internet]. 2015 [cited 2021 Apr 16] ; 18(3): 507-519. Available from: http://www.scielo.br/scielo.php?script=sci_arttext\&pid=S1809-98232015000300507\&lng=en.

24. Aguiar ACSA, Menezes TMO, Camargo CL. Significado do cuidar de pessoas idosas sob a ótica do familiar: um estudo interacionista simbólico. Rev Min Enferm. [Internet] 2017[citado 2021 Abril 25];21(e-1004). Disponivel em: http://www.reme.org. br/artigo/detalhes/1140.

25. Ribeiro PCC, Almada DSQ, Souto JF, Lourenço RA. Permanence in the labour market and life satisfaction in old age. Ciênc. saúde coletiva [Internet]. 2018 [cited 2021 Apr16]; 23(8): 2683-2692. Available from: https://doi.org/10.1590/141381232018238.20452016.

26. Aerosa SVG, Araújo CK, Cardoso CMC, Moreira EP. Envelhecimento: relações pessoais e familiares. Barbarói, Santa Cruz do Sul [Internet] 2012 [cited 2021 Apr 16]; 36(ed. esp.):120-132. Disponível em: https://online.unisc.br/seer/index.php/barbaroi/ article/viewFile/2931/2126

27. Barricelli ILFOBL, Sakumoto IKY, Silva LHM, Araújo CV. Influência da orientação religiosa na qualidade de vida de idosos ativos. Rev. bras. geriatr. gerontol. [Internet]. 2012 [citado 2021 Abr 16];15(3): 505-515. Disponivel em: http://www.scielo.br/scielo. php?script=sci_arttext\&pid=S1809-98232012000300011\&I$\mathrm{ng}=\mathrm{pt}$.

28. Confortin SC, Giehl MWC, Antes DL, Schneider IJC, d'Orsi E. Positive self-rated health in the elderly: a population-based study in the South of Brazil. Cad. Saúde Pública [Internet]. 2015 [cited 2021 Apr 16]; 31(5):1049-1060. Available from: http://www.scielo.br/scielo.php?script=sci_arttext\&pid=S0102-311X2015000500016\&lng=en.

29. Borim FSA, Barros MBA, Neri AL. Autoavaliação da saúde em idosos: pesquisa de base populacional no Município de Campinas, São Paulo, Brasil. Cad. Saúde Pública [Internet]. 2012 [citado 2021 Abril 16]; 28(4):769-780. Available from: http://www.scielo.br/scielo.php?script=sci_arttext\&pid=S0102-311X2012000400016\&lng=en.

30. Pilger C, Menon MU, Mathias TAF. Health services use among elderly people living in the community. Rev. esc. enferm. USP
[Internet].2013 [cited 2021 Apr 16]; 47(1): 213-220. Available from: http://www.scielo.br/scielo.php?script=sci_arttext\&pid=S0080-62342013000100027\&lng=en.

31. Confortin SC, Giehl MWC, Antes DL, Schneider IJC, d'Orsi $\mathrm{E}$. Positive self-rated health in the elderly: a population-based study in the South of Brazil. Cad. Saúde Pública [Internet]. 2015 [cited 2021 Apr 16]; 31(5):1049-1060. Available from: http://www.scielo.br/scielo.php?script=sci_arttext\&pid=S0102-311X2015000500016\&lng=en.

32. Borim FSA, Barros MBA, Neri AL. Autoavaliação da saúde em idosos: pesquisa de base populacional no Município de Campinas, São Paulo, Brasil. Cad. Saúde Pública [Internet]. 2012 [citado 2021 Abril 16]; 28(4):769-780. Available from: http://www.scielo.br/scielo.php?script=sci_arttext\&pid=S0102-311X2012000400016\&lng=en.

33. Busato MA, Gallina LS, Teo CRPA, Ferreti F, Pozzagnol M. Autopercepção de saúde e vulnerabilidade em idosos. Revista Baiana de Saúde Pública [Internet] 2014 [citado 2021 Abril 16]; 38(3): 625-635. Disponivel em: https://www.researchgate.net/ profile/Carla_Rosane_Teo/publication/280979027_Autopercepcao_de_saude_e_vulnerabilidade_em_idosos/links/55cf829708ae502646ä44e4.pdf.

34. Hagger-Johnson G, Carr E, Murray E, Stansfeld S, Shelton N, Stafford M, Head J. (2017). Association between midlife health behaviours and transitions out of employment from midlife to early old age: Whitehall II cohort study. BMC Public Health [Internet] 2017 [cited $2021 \mathrm{Apr} 16$ ];17(1), 82. Available from: https:// pubmed.ncbi.nlm.nih.gov/28095887/.

35. Choi E, Tang F, Kim S, Turk P. Longitudinal relationships between productive activities and functional health in later years: $A$ multivariate latent growth curve modeling approach. Int J Aging Hum Dev [Internet] 2016 [cited $2021 \mathrm{Apr}$ 16];83(4):418-440. Available from: https://pubmed.ncbi.nlm.nih.gov/27461262/.

36. Mazzonna F, Peracchi F. Aging, Cognitive Abilities and Retirement. European Economic Review. [Internet] 2012 [cited 2021 Apr 16];56(4):691-710. Available from: https://www.sciencedirect.com/science/article/pii/S0014292112000463.

37. Caban-Martinez AJ, Lee DJ, Fleming LE, Tancredi DJ, Arheart KL, LeBlanc WG et al. Arthritis, occupational class, and the aging US workforce. Am J Public Health [Internet] 2011 [cited $2021 \mathrm{Apr}$ 16]; 101(9): 1729-1734. Available from: https://www.ncbi.nlm. nih.gov/pmc/articles/PMC3154222/.

38. Cintra TS, Ribeiro DF, Andrade AS. O cotidiano de aposentados que continuam trabalhando de maneira informal na indústria calçadista: percepções sobre a aposentadoria e o trabalho atual. Cad. psicol. soc. trab. [Internet] 2010 [citado 2021 Abril 16]; 13(2), 277-287. Disponivel em: http://pepsic.bvsalud.org/pdf/cpst/ v13n2/v13n2a09.pdf.

39. Marra AV, Souza MMP, Marques AL, Melo MCOL. Significado do trabalho e envelhecimento. Revista Administração em Diálogo. [Internet] 2013 [citado 2021 Abril 16];15(2): 103-128. Disponivel em: https://revistas.pucsp.br/index.php/rad/article/ view/12737. 